\title{
Chronic Intestinal Pseudo-obstruction: Clinical and Manometric Characteristics in the Chilean Population
}

\author{
Edith Pérez de Arce, ${ }^{1}$ Glauben Landskron, ${ }^{1}$ Sandra Hirsch, ${ }^{2}$ Carlos Defilippi, ${ }^{1}$ and Ana María Madrid ${ }^{1 *}$ \\ ${ }^{1}$ Laboratory of Functional Digestive Diseases and Motility, Section of Gastroenterology, Clinical Hospital Universidad de Chile, Santiago, Chile; \\ ${ }^{2}$ Laboratory Aging and ECRAN, Human Nutrition Unit, Institute of Nutrition and Food Technology (INTA), Universidad de Chile, Santiago, Chile
}

\section{Background/Aims}

Chronic intestinal pseudo-obstruction (CIPO) is a rare syndrome characterized by a failure of the propulsion of intraluminal contents and recurrent symptoms of partial bowel obstruction in the absence of mechanical obstruction. Regional variations of the intestinal compromise have been described. Intestinal manometry can indicate the pathophysiology and prognosis. Our objective is to establish the demographic and clinical characteristics of group Chilean patients and analyze the motility of the small intestine and its prognostic value.

\section{Methods}

Patients with symptoms of intestinal pseudo-obstruction with dilated bowel loops were included, in all of whom a manometry of the small intestine was performed using perfused catheters.

\section{Results}

Of the 64 patients included, 51 women (average age $41.5 \pm 17.6$ years), 54 primary and 10 secondary CIPO were included. Dilatation of the small intestine was the only finding in 38 patients; in the remaining, the compromise was associated with other segments, primarily the colon. Forty-nine patients underwent 65 surgeries, mainly exploratory laparotomies and colectomies. Intestinal manometry was performed on all patients; 4 "patterns" were observed: neuropathic $(n=26)$, myopathic $(n=3)$, mixed $(n=24)$, and a group without motor activity $(n=11)$. The most relevant findings were the complex migrating motor disorders and decreased frequency and propagation of contractions. The 9 patients who died had a severe myopathic compromise.

\section{Conclusions}

In our series, isolated small bowel compromise was the most common disorder. Neuropathic motor compromise was observed in most of the patients. Mortality was associated with severe myopathic compromise.

(J Neurogastroenterol Motil 2017;23:273-280)

\section{Key Words}

Intestinal pseudo-obstruction; Manometry, intestinal obstruction; Myoelectric complex; Small intestine

\section{Received: June 24, 2016 Revised: August 13, 2016 Accepted: August 21, 2016}

(a) This is an Open Access article distributed under the terms of the Creative Commons Attribution Non-Commercial License (http://creativecommons. org/licenses/by-nc/4.0) which permits unrestricted non-commercial use, distribution, and reproduction in any medium, provided the original work is properly cited.

*Correspondence: Ana María Madrid, MD Laboratory of Functional Digestive Diseases and Motility, Section of Gastroenterology, Clinical Hospital Universidad de Chile, Santos Dumont 999, Independencia, Santiago 8380456, Chile Tel: +56-2-29788350, E-mail: anamariamadrid@gmail.com 


\section{Introduction}

Chronic intestinal pseudo-obstruction (CIPO) is a rare syndrome that can affect all segments of the gastrointestinal tract. It is characterized by a failure of the propulsion of intraluminal contents due to the inability to generate a suitable motor "pattern," either due to a deficit of the contractile force or of the coordination of the waves. $^{1-5}$

Clinically there are recurrent episodes characterized by symptoms, clinical signs, and images of partial bowel obstruction in the absence of a mechanical obstruction, which often lead to performing unnecessary surgeries. The clinical picture usually has a progressive nature, in which important abdominal symptoms appear, such as pain, bloating, and distension, associated with alterations of intestinal transit and weight loss. ${ }^{1-5}$

Frequently the presence of intestinal bacterial overgrowth is suspected and the patients are treated with antibiotics, although this phenomenon has rarely been studied systematically. ${ }^{6,7}$ The progression of the illness is usually associated with malabsorption and malnutrition, sometimes becoming necessary to resort to parenteral nutrition. ${ }^{8,9}$

With regard to the pathophysiology the compromise of intrinsic and extrinsic neural structures, muscles, Cajal cells, or a combination of these have been described. These alterations can be idiopathic when they appear in the absence of other disorders, or may be secondary to a number of different conditions that include neurological, endocrine, metabolic, autoimmune, paraneoplastic syndrome, and infectious diseases. Most cases are often sporadic, although some forms have been described as related to genetic alterations and others as family-related in which the genetic alteration has not been identified. ${ }^{2}$

The diagnosis is usually difficult and late to establish. Recently, some authors ${ }^{10}$ have proposed basic diagnostic criteria that include intestinal obstruction symptoms, abdominal pain and bloating, bowel dilatation or fluid levels established by different methods, and the absence of structural changes explaining the dilated loops.

The incidence and prevalence of CIPO is unknown. Mostly, the data come from small case series. A Japanese study estimated a prevalence of 1.0 versus 0.8 cases per 100000 inhabitants in men and women respectively. ${ }^{11}$ Western studies have shown a higher frequency of CIPO in females, that ranges from $64 \%$ to $68 \%$. $^{9,12}$

Intestinal manometry, not always available in all centers, has been considered to be an important contribution to the diagnosis of the syndrome, but the diagnosis cannot rely solely on the study of motility with manometry. It can also contribute to establishing the pathophysiology of the underlying dysmotility: in the presence of contractions of normal amplitude, but which is uncoordinated, it is possible to assume that the intestinal muscle is normal and that the neurogenic control is altered. In contrast, the presence of contractions of low amplitude with normal coordination suggests an alteration of the myopathic character, but, of course, considering that the dilatation of the bowel loops can be the cause of low amplitude waves. Some studies suggest that intestinal manometry ${ }^{12}$ and even the esophageal ${ }^{13}$ may also be useful as a prognostic factor of the course of the pathology.

Our center's Motility laboratory has been conducting intestinal motility studies on a regular basis for 3 decades, which has permitted us to gather a wide number of cases of patients with CIPO. The aims of these studies were to establish the demographic characteristics of our cases, clinical manifestations, treatment, and evolution, and to analyze the results of the manometric studies and gauge their value to predict the evolution of the clinical picture.

\section{Materials and Methods}

\section{Patients}

A descriptive, retrospective study was carried out on 64 patients whose clinical picture was compatible with CIPO during the period between 1995 and 2014. These patients met the following diagnostic criteria for CIPO to be included in the study: one or more suggestive episodes of intestinal obstruction in the absence of evidence of mechanical obstruction. This was demonstrated by imaging or surgery, dilation of the small bowel loops evidenced by some method of imaging in the critical period (radiology, ultrasound and/ or abdominal computed tomography, and enteroclysis computed tomography) being able to coexist with dilation of other segments of the digestive tract. In radiological studies, the definition of the dilation loop in the small bowel is when the loop shows a diameter of greater than $3 \mathrm{~cm}$.

Eight patients who had a history of multiple abdominal surgeries, some of which predated the onset of intestinal obstruction, were excluded.

Socio-demographic data were registered on each patient: medical and surgical history, symptom onset and progression of symptoms, number of attacks, treatments received, and evaluation of the laboratory tests, images, operational protocols, and intestinal biopsies and reports of surgeries, if they were available. According to medical history, patients were classified as primary or idiopathic 
CIPO (if an underlying cause of the disease was not identified) and as secondary CIPO (with an identifiable cause of the motility disorder). Forty-seven patients were evaluated, treated and followed in our institution over the whole period by one of the authors and the remaining 17 were followed in other centers, from which appropriate information concerning their evolution was obtained.

\section{Intestinal Manometry: Motility Studies}

Small bowel manometry was performed on all patients during an inter-crisis period and was studied by means of a multilumen perfused catheter assembly, which incorporated 4 polyvinyl tubes (internal diameter, $0.9 \mathrm{~mm}$ ) glued together with tetrahydrofuran and with side holes spaced $3 \mathrm{~cm}$ from each other. They were continuously infused with bubble free distilled water with a pneumohydraulic capillary infusion system (Arndorfer Medical Specialities, Greendale, Wisconsin, USA) at a rate of $0.4 \mathrm{~mL} / \mathrm{min}$.

The manometric catheters were attached to external TP-400t pressure transducers and connected to a Nihon Kohden polygraph (Nihon Kohden, Tokyo, Japan), registering the waves on a paper record. The outputs of the amplifiers were, in turn, connected to an analog-digital converter in a personal computer, and signals were analyzed by a computer program developed in our laboratory. After an 8 hour overnight fast, the catheter assembly was passed into the mouth to the stomach and was placed under fluoroscopic control, leaving the distal hole at $10 \mathrm{~cm}$ past the angle of Treitz. The studies were conducted for at least 5 hours during the "fasting period."

\section{Analysis of motor activity}

The different Phases of the migrating motor complex (MMC) and clustered contractions (CC) were identified by direct visual inspection of the chart-recording paper using the following criteria: Phase I was characterized by the complete absence of contractions; Phase II consisted of irregular phasic contractions, which culminated in a burst of rhythmic phasic contractions at a frequency of 10 to $12 \mathrm{cpm}$ with a minimum duration of 3 minutes that appear following a Phase II (Phase III). To define MMC activity, the presence of at least one Phase III, preceded by Phase II and followed by Phase I, was required. Clustered contractions were defined as a sequence of 3 to 10 rhythmic contractions, preceded and followed by a quiescent period of 1 minute to 5 minutes in duration. The presence of more than $2 \mathrm{CC}$ per hour was considered abnormal. The duration of Phase I, Phase II, and Phase III and duration of the MMC were calculated when they were present. The frequency and amplitude of contractions and the percentage of waves propagated were calculated through the automated system. An intestinal motil- ity index (IMI) was calculated with the product of the contractions frequency and mean amplitude. ${ }^{14}$

Normal values were based on previous studies performed on normal subjects in our laboratory. A neuropathic compromise was defined as the absence of Phase III or Phase III with a shorter duration than 3 minutes or longer than 15 minutes, the absence of propagation, or retrograde propagation, decreased frequency of the contractions greater than 2 standard deviations from the mean value of the control group in the presence of a normal amplitude. ${ }^{14}$

Compromise was defined as myopathic if decreased amplitude of contractions were found to be greater than 2 standard deviations below the average. Joint compromise was defined as the association of both disorders. The absence of contractions during the recording period was independently analyzed.

\section{Histology}

The reports available on transmural biopsies of the small intestine obtained in surgeries performed during periods of crisis were reviewed.

\section{Ethics}

This research project was approved by the Scientific Ethics Committee of the Clinical Hospital Universidad de Chile.

\section{Statistical Methods}

The data obtained were entered into a database created in the software Microsoft Excel. The data were analyzed using the statistical software EpiInfo 7 (CDC, Atlanta, GA, USA), using the ANOVA test for the continuous variables and Chi-square test or Fisher exact test, as deemed appropriate, for the discrete variables.

\section{Results}

\section{Clinical Characteristics}

The average age of the patients when diagnosed was $41.6 \pm$ 17.6 years (13-76 years range), 51 patients (79.7\%) were female. Fifty-four patients were classified as primary CIPO, 2 of whom were brothers and had the same manometric pattern. Of the 10 patients with secondary CIPO, 9 were female. Eight patients presented symptoms compatible with systemic sclerosis and 2 with systemic lupus erythematosus.

The symptoms began before the age of 15 in 21 patients. The CIPO diagnosis was established at about $12.7 \pm 1.5$ years after the first episode of intestinal sub-occlusion, and patients presented on 
an average 2.4 episodes of sub-occlusion before the diagnosis was established. Symptoms and their prevalence in the inter-crisis periods are shown in Table 1. Intestinal transit disorders, bloating, and abdominal pain were the most relevant symptoms.

\section{Imaging Studies}

Imaging studies showed dilatation of small bowel loops (Fig. 1) in one or more tests, most of which were plain abdominal X-rays and computerized abdominal tomography (Table 2). The dilatation of the small bowel as the only finding was observed in 38 patients, and in the others, the tests revealed another compromised segment of the digestive tract, mainly the colon (Table 3).

\section{Surgery}

Forty-nine patients underwent surgery with a total of 65 surgical interventions prior to the CIPO diagnosis. Nineteen patients had 1 surgery, 12 patients had 2, and 8 patients had 3 or more interventions. In 15 patients, 30 exploratory laparotomies were performed during crises of pseudo-obstruction, in which a mechanical

Table 1. Frequency of Symptoms and Their Prevalence in the Intercrisis Periods

\begin{tabular}{lc}
\hline \multicolumn{1}{c}{ Symptoms } & $\mathrm{n}(\%)$ \\
\hline Constipation & $32(50.0)$ \\
Diarrhea & $28(43.8)$ \\
Abdominal distension & $40(62.5)$ \\
Abdominal pain & $33(51.6)$ \\
Vomiting & $21(32.8)$ \\
Weight loss & $19(29.7)$ \\
\hline
\end{tabular}

Intestinal transit disorders (Constipation and diarrhea), bloating, and abdominal pain were the most relevant symptoms on patients with chronic intestinal pseudo-obstruction (CIPO).

" $\mathrm{n}$ " is the number of patients for each of the most common symptoms. The percentage corresponds to the number of patients with symptoms of the number of patients diagnosed with CIPO $(n=64)$.

Table 2. Image Studies That Showed Dilatation of Small Bowel Loops

\begin{tabular}{lc}
\hline \multicolumn{1}{c}{ Tests } & $\mathrm{n}(\%)$ \\
\hline Simple abdominal radiography & $45(70.3)$ \\
Abdominal computed tomography & $31(48.4)$ \\
Enteroclysis computed tomography & $7(10.9)$ \\
Ultrasound & $6(9.4)$ \\
\hline
\end{tabular}

Radiological examinations reveal dilated small bowel loops. In 45,13 , and 6 patients 1,2 , and 3 tests respectively.

" $\mathrm{n}$ " is number of patients with that specific test. The percentage corresponds to the number of patients with specific test of the number of patients diagnosed with chronic intestinal pseudo-obstruction $(\mathrm{n}=64)$. obstruction was ruled out. Adhesions were found in 6 and of these patients, 3 presented with volvulus ( 2 small intestine and 1 colon), with resection performed in 2. Eleven patients underwent total colectomy and 8 underwent hemicolectomy for severe constipation, 2 of whom initially underwent partial colectomy followed by total colectomy. Two patients underwent hemigastrectomy and 4 received anti-reflux surgery. One patient underwent gastrostomy and another underwent jejunostomy as part of the treatment. Twelve patients also had a history of cholecystectomy and 8 had gynecological surgery.

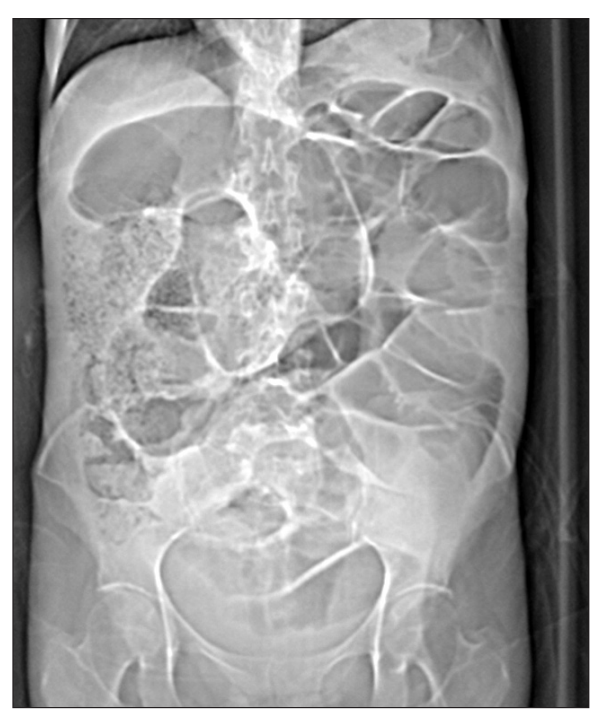

Figure 1. Plain abdominal X-ray of a patient with dilated loop of the small intestine during a crises period of chronic intestinal pseudo-obstruction (CIPO). Plain abdominal X-rays corresponds to a woman, 48 years old, diagnosed with CIPO. It is noted that only dilated loops of small intestine are seen. Currently, the patient is deceased.

Table 3. Compromise of the Small Intestinal and Other Segments of the Digestive Tract

\begin{tabular}{lc}
\hline \multicolumn{1}{c}{ Segments of the digestive tract } & $\mathrm{n}(\%)$ \\
\hline Small intestine only & $38(59.4)$ \\
Small intestinal + colon & $22(34.4)$ \\
Small intestinal + stomach + esophagus & $2(3.1)$ \\
Stomach + small intestine & $1(1.6)$ \\
Stomach + small intestine + colon & $1(1.6)$ \\
\hline
\end{tabular}

The dilatation of the small bowel as the only finding was observed in 38 patients, and in other 26 patients the tests revealed another compromised segment of the digestive tract, mainly the colon.

" $\mathrm{n}$ " is corresponding number of patients with compromise in different segments of the digestive tract. The percentage corresponds to the number of patients with compromise in segment of the digestive tract of the number of patients diagnosed with chronic intestinal pseudo-obstruction $(n=64)$. 


\section{Intestinal Manometry}

There were 68 studies undertaken on the small intestinal motility; in 4 patients, 2 studies were carried out separately for a period of over a year and no significant changes were observed between the 2 manometric studies.

The average duration of the studies was $304 \pm 28$ minutes. Most of the patients presented a neuropathic "pattern." In 26 patients this pattern was presented as the only alteration and in 24 patients and it appeared to be associated with myopathic compromise ("Mixed pattern"), while in 3 patients, only myopathic compromise was observed. Eleven patients with complete absence of contractions during the entire recording period were grouped separately from the patients with other disorders. Table 4 shows the main features of the various motility parameters analyzed.

The MMC was absent in $61.5 \%$ of the patients classified as neuropathic and $71.0 \%$ in the mixed group. The number of Phase III per hour was lower among patients in the neuropathic and mixed groups when compared to the control group.

The total number of Phase III was diminished in the neuropathic as in the mixed groups, and most of the Phase III that were registered presented abnormal characteristics regarding propagation and duration. The contraction frequency was reduced in the mixed and myopathic groups compared to the control group. The percentage of the waves propagated in patients with CIPO was found to be markedly reduced to less than $1 \%$, with $25 \%$ in the control group. An increase in the clustered contractions was registered especially in the neuropathic group. The strength of contractions represented by the amplitude of the waves was found diminished in the mixed and myopathic groups. On the other hand, values in the neuropathic group were no different from the normal control group. The IMI was lower in all 3 groups when compared with the control group values.

\section{Histology}

Transmural biopsies were performed in only 5 patients, and which reported agangliosis in 1 patient and hypoganglionosis in 4 .

\section{Medical Treatment}

The drug treatment was based on the different prokinetics and antibiotics shown in Table 5. In nutrition management, 15 patients required parenteral nutrition, 12 were hospitalized during some of the crises while 3 were ambulatory, 4 received enteral tube feeding (one patient for jejunostomy), and 1 patient was fed by gastrostomy.

Table 5. Pharmacotherapy

\begin{tabular}{lc}
\hline Pharmaceutic & $\mathrm{n}(\%)$ \\
\hline Domperidone & $44(68.8)$ \\
Cisapride & $23(35.9)$ \\
Erythromycin & $19(29.7)$ \\
Levosulpiride & $11(17.2)$ \\
Metoclopramide & $3(4.7)$ \\
Neostigmine & $2(3.1)$ \\
Tegaserod & $2(3.1)$ \\
Antibiotics & $21(32.8)$ \\
\hline
\end{tabular}

" $\mathrm{n}$ " is corresponding number of patients for each drug. The percentage corresponds to the number of patients with medication of the number of patients diagnosed with chronic intestinal pseudo-obstruction $(n=64)$.

Table 4. Manometric Findings of the Different Motility Patterns in Patients with Chronic Intestinal Pseudo-obstruction Compared to Controls

\begin{tabular}{|c|c|c|c|c|c|}
\hline \multirow{2}{*}{ Manometric findings } & \multicolumn{3}{|c|}{ Pattern } & \multirow{2}{*}{$\begin{array}{l}\text { Absent activity } \\
\text { motor }\end{array}$} & \multirow{2}{*}{$\begin{array}{l}\text { Control } \\
\text { group }\end{array}$} \\
\hline & Neuropathic & Myopathic & Mixed & & \\
\hline Number of patients & 26 & 3 & 24 & 11 & 20 \\
\hline Absent cyclic activity $(\%)$ & 61.5 & 0 & 71.0 & 100 & 0 \\
\hline Number Phase III per hour & $0.03 \pm 0.04^{\mathrm{a}}$ & $0.53 \pm 0.13$ & $0.20 \pm 0.03^{\mathrm{a}}$ & 0 & $0.50 \pm 0.04$ \\
\hline Number abnormal Phase III/total Phase III per hour & $18 / 20$ & $0 / 3$ & $15 / 16$ & 0 & $0 / 61$ \\
\hline Frequency of contractions per minute (cpm) & $0.70 \pm 0.09^{\mathrm{a}}$ & $0.28 \pm 0.04$ & $0.33 \pm 0.05^{\mathrm{a}}$ & 0 & $1.20 \pm 0.06$ \\
\hline$\%$ of propagated contractions & $0.30 \pm 0.10$ & $0.31 \pm 0.31$ & $0.44 \pm 0.14$ & 0 & $25.00 \pm 15.00$ \\
\hline Clustered contractions per hour & $3.90 \pm 0.80^{\mathrm{a}}$ & $2.00 \pm 1.30$ & $0.70 \pm 0.30$ & 0 & $1.20 \pm 0.10$ \\
\hline Mean amplitude of contractions ( $\mathrm{mmHg}$ ) & $27.10 \pm 1.90$ & $16.00 \pm 2.80^{\mathrm{a}}$ & $15.60 \pm 0.90^{\mathrm{a}}$ & 0 & $31.00 \pm 6.70$ \\
\hline IMI & $20.30 \pm 3.00^{\mathrm{a}}$ & $4.50 \pm 1.30^{\mathrm{a}}$ & $5.80 \pm 1.10^{\mathrm{a}}$ & 0 & $38.40 \pm 12.60$ \\
\hline
\end{tabular}

$\%$ of propagated contraction, percentage contraction waves propagated with respect to the sum of all waves that occurred in the period registered; IMI, intestinal motility index.

${ }^{a} P<0.01$ compared to control group. 


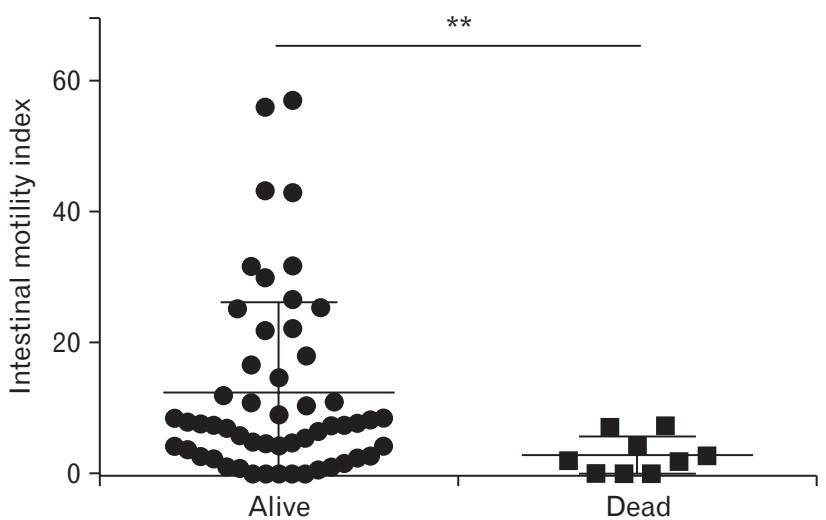

Figure 2. Intestinal motility index (IMI) on alive and dead patients diagnosed with chronic intestinal pseudo-obstruction. The IMI corresponded to the product of the contractions frequency and mean amplitude. In comparing the manometric parameters of the patients who died with alive patients, IMI was $2.8 \pm 0.9$ vs $12.1 \pm 1.8(* * P=$ 0.007). The 9 patients who died had a severe myopathic compromise.

\section{Mortality}

Nine patients died during the follow-up period, the causes of which were: in 3 patients, the evolution of the fundamental disorder, in 2 cases sepsis by catheter feeding, in 2 others during a pseudoobstruction crisis, and in the other 2 the cause of death was not established.

A relationship between mortality and intestinal manometry was established: 3 deceased patients presented a pattern of intestinal motility without any contractions; 5 belonged to the mixed group, and 1 to the myopathic group. In comparing the manometric parameters of the patients who died with the others, the amplitude of the contractions observed was found to be $10.6 \pm 2.7 \mathrm{mmHg}$ vs 19.5 $\pm 1.5 \mathrm{mmHg}(P<0.02)$. The IMI was $2.8 \pm 0.9$ vs $12.1 \pm 1.8(P$ $=0.007$ ) (Fig. 2).

\section{Discussion}

Although CIPO is an infrequent clinical condition, over the years our center has managed to gather together a comparatively significant number of cases. Thus, we have been able to establish the clinical characteristics and evaluate the evolution of a group of adult patients and, in particular, we have analyzed the role of intestinal manometry in the diagnosis and prognosis of the syndrome. As in other studies with a similar number of patients, a predominance of females was observed, and most of the cases corresponded to primary CIPO., ${ }^{410,12,13}$

The average age of our patients at diagnosis was similar to that described in European studies ${ }^{12}$ and significantly younger than the age observed in Japanese studies. This is probably due to enrollment of patients with colonic involvement, which appear at an older age. .0,11 $^{11}$

The frequency with which the main symptoms appeared is similar to that observed in other studies; however, in our study, as in others, their intensity was not recorded. Thus, one might speculate that since pain is a very common symptom, its intensity may have been less than that described in the literature, since painkillers are not listed among the drugs received by the patients. Instead, the use of prokinetics predominates.

In the imaging studies, the disorder that appeared most frequently was isolated small bowel compromise, followed by the combination of compromised small intestine and colon. In the work by Masaki et al, ${ }^{4}$ the distribution was reversed with the colon appearing compromised most frequently, and the least common disorder being the small intestine.

As described in other series ${ }^{4,12}$ our patients underwent numerous surgeries, an important number of which were motivated by clinical suspicion of intestinal obstruction and before the diagnosis of CIPO. In these conditions, it may be difficult to suspect the diagnosis and surgery may be justified. Small bowel resections were conducted only in the presence of volvulus and infeasibility of the intestinal loop. On other hand, a relatively large number of colectomies were performed as treatment for chronic constipation. In these patients there was no suspicion of a possible diffuse motility disorder, which could have been determined by the intestinal manometry. Our group studied 43 patients with severe constipation and we found that small bowel motility was altered in $33 .^{15}$

We do not know what the impact of surgery was on the patient's outcome. A study showed that the results of surgical treatment were better in the presence of isolated colon compromise and less effective in the presence of both compromised small intestine and colon. ${ }^{4}$ Some authors ${ }^{16}$ have analyzed the evolution of a large group of patients with CIPO undergoing non-transplant surgery, observing that surgical treatment was associated with high mortality (7.9\%) and morbidity (58.2\%); the latter associated with intraoperative intestinal lesions, idiopathic CIPO, and emergency surgery, and concluded that efforts should be made to avoid surgery, if possible.

Findings of intestinal manometry in children and adults in general have been variable. ${ }^{17}$ Small bowel manometry in our study showed neuropathic compromise in $78 \%$ of patients, either alone or associated with a decreased amplitude of contractions. In contrast, the isolated contractile force compromise was uncommon. We also 
observed a group of patients in whom all motor activity was absent during the registration. Our results are intermediate in nature compared to other studies which have also studied intestinal motility in CIPO. While in one of them ${ }^{18}$ only neuropathic disorders were mentioned, motility alterations have been found in $60 \%$ of other reports. $^{12,13}$ The hypomotility varied between $20 \%$ and $79 \%$. It is possible that these differences reside in the characteristics of patients or could be related to the criteria which were cataloged the motor disorders. The compromise of the motor activity in our patients in general was very extensive. The MMC was absent between $60 \%$ and $70 \%$ depending on the group, the Phase III was quantitatively reduced and when they were present, most presented an abnormal character

One of the main findings was a very severe reduction of the prolonged phasic waves, which clearly explains the deterioration of endoluminal content propulsive ability. Manometric findings were associated with mortality since a third of the patients who died presented a "pattern" of motility characterized by absence of contractions, and the rest a severe disorder of a myopathic character. A similar observation has been made by other authors ${ }^{12}$ so that in the presence of a severe myopathic disorder, patients should be monitored and managed more thoroughly.

Despite the rareness of this syndrome, our study gathered a significant number of patients, whose clinical characteristics were similar to those described in Europe and USA, reflecting the difficulties related to the diagnosis, which generally came late and after several surgeries. Intestinal manometry revealed a severe overall compromise and the myopathic "pattern" associated with mortality. The clinical data of CIPO should be better recognized and understood by the medical community to make use of all the resources available for confirmation whenever it is suspected.

Acknowledgements: The investigators would like to thank $\mathrm{Mr}$ José Matus for their collaboration in the carrying out the manometries.

\section{Financial support: None.}

\section{Conflicts of interest: None.}

Author contributions: Edith Pérez de Arce and Glauben Landskron: analysis and interpretation of data and drafting the article; Sandra Hirsh: analysis and interpretation of data; Carlos Defilippi: revising it critically for important intellectual content; and Ana María Madrid: conception and design, analysis and interpre- tation of data, drafting the article, revising it critically for important intellectual content, and final approval of the version to be published.

\section{References}

1. Lauro A, De Giorgio R, Pina AD. Advancement in the clinical management of intestinal pseudo-obstruction. Expert Rev Gastroenterol Hepatol 2015;9:197-208

2. De Giorgio R, Cogliandro RF, Barbara G, Corinaldesi R. Stanghellini V. Chronic intestinal pseudo-obstruction: clinical features, diagnosis, and therapy. Gastroenterol Clin North Am 2011;40:787-807.

3. Joly F, Amiot A, Coffin B, Lavergne-Slove A, Messing B, Bouhnik Y. [Pseudo-obstruction intestinale chronique.] Gastroenterol Clin Biol 2006;30:975-985. [French]

4. Masaki T, Sugihara K, Nakajima A, Muto T. Nationwide survey on adult type chronic intestinal pseudo-obstruction in surgical institutions in Japan. Surg Today 2012;42:264-271.

5. Schuffler MD, Rohrmann CA, Chaffee RG, Brand DL, Delaney JH, Young JH. Chronic intestinal pseudo-obstruction: a report of 27 cases and review of the literature. Medicine 1981;60:173-196.

6. Pearson AJ, Brzechwa-Ajdukiewicz A, McCarthy CF. Intestinal pseudoobstruction with bacterial overgrowth in the small intestine. Am J Dig Dis 1969;14:200-205.

7. Pérez de Arce E, Defilippi C, Madrid AM. [Sobrecrecimiento bacteriano intestinal y pseudoobstrucción intestinal crónica. Una relación poco conocida.] Gastroenterol latinoam 2014;25:257-263.[Spanish]

8. Gabbard SL, Lacy BE. Chronic intestinal pseudo-obstruction. Nutr Clin Pract 2013;28:307-316.

9. Amiot A, Joly F, Alves A, Panis Y, Bouhnik Y, Messing B. Long-term outcome of chronic intestinal pseudo-obstruction adult patients requiring home parenteral nutrition. Am J Gastroenterol 2009;104:1263-1270.

10. Onkubo H, Lida L, Takahashi H, et al. An epidemiologic survey of chronic intestinal pseudo-obstruction and evaluation of the newly proposed diagnostic criteria. Digestion 2012;86:12-19.

11. Lida H, Ohkubo H, Inamori M, Nakajima A, Sato H. Epidemiology and clinical experience of chronic intestinal pseudo-obstruction in Japan: a nationwide epidemiologic survey. J Epidemiol 2013;23:288-294.

12. Stanghellini V, Cogliandro RF, De Giorgio R, et al. Natural history of chronic idiopathic intestinal pseudo-obstruction in adults: a single center study. Clin Gastroenterol Hepatol 2005;3:449-458.

13. Amiot A, Joly F, Cazals-Hatem D, et al. Prognostic yield of esophageal manometry in chronic intestinal pseudo-obstruction: a retrospective cohort of 116 adult patients. Neurogastroenterol Motil 2012;24:1008, e542.

14. Madrid, AM, Poniachik J, Quera R, Defilippi C. Small intestinal clustered contractions and bacterial overgrowth: a frequent finding in obese patients. Dig Dis Sci 2011;56:155-160.

15. Madrid AM, Defilippi C. [Disturbances of small intestinal motility in patients with chronic constipation.] Rev Med Chil 2006;134:181-186. [Spanish] 
16. Sabbagh C, Amiot A, Maggiori L, Corcos O, Joly F, Panis Y. Nontransplantation surgical approach for chronic intestinal pseudo-obstruction: analysis of 63 adult consecutive cases. Neurogastroenterol Motil 2013;25:e680-e686.

17. Stanghellini V, Cogliandro RF, De Giorgio R, Barbara G, Salvioli B, Corinaldesi R. Chronic intestinal pseudo-obstruction: manifestations, nat- ural history and management. Neurogastroenterol Motil 2007;19:440452.

18. Stanghellini V, Camilleri M, Malagelada JR. Chronic idiopathic intestinal pseudo-obstruction: clinical and intestinal manometric findings. Gut 1978;28:5-12. 